- Original Article

\title{
Family Functioning and Communication in Spouses of Patients with Parkinsonism
}

\author{
Seo Young Kang', Myung Hwa Yang', Jung Ah Lee', Wooyoung Jang'2, Chong Sik Lee, Young Sik Kim,* \\ 'Department of Family Medicine, Asan Medical Center, University of Ulsan College of Medicine, Seoul, Korea \\ ${ }^{2}$ Department of Neurology, Gangneung Asan Hospital, University of Ulsan College of Medicine, Gangneung, Korea \\ ${ }^{3}$ Department of Neurology, Asan Medical Center, University of Ulsan College of Medicine, Seoul, Korea
}

\begin{abstract}
Background: Patients with parkinsonism exhibit motor symptoms, cognitive impairment, and neuropsychiatric changes, and these symptoms increase caregiver burden. Family dynamics can be influenced by the presence of comorbidities, which is especially important in diseases causing caregiver burden. We investigated the effects of spousal parkinsonism on family functioning and communication.

Methods: Couples without parkinsonism, who visited hospital-based family practices, were recruited by 28 family physicians from 22 hospitals between April 2009 and June 2011; patients with parkinsonism and their spouses were recruited from a single institution. The participants completed questionnaires on demographic characteristics, lifestyle factors, family functioning (the Korean version of the Family Adaptation and Cohesion Evaluation Scale [FACES] III), and family communication (the Family Communication Scale of the FACES-IV). We compared family functioning and communication between spouses of the patients with and without parkinsonism.

Results: The mean family adaptability and cohesion scores of the spouses of the patients with parkinsonism were $23.09 \pm 6.48$ and $32.40 \pm 8.43$, respectively, whereas those of the control group were $23.84 \pm 5.88$ and $34.89 \pm 7.59$, respectively. Family functioning and family communication were significantly different between the spouses of individuals with and without parkinsonism. After adjusting for age, sex, income, and cardiovascular disease in the logistic regression analysis, family functioning was found to significantly deteriorate in the spouses of patients with parkinsonism but not the control group. Family communication decreased significantly in spouses of patients with parkinsonism.

Conclusion: Family functioning and family communication significantly deteriorated in spouses of patients with parkinsonism.
\end{abstract}

Keywords: Parkinsonism; Family Dynamics; Spouses; Caregivers 


\section{INTRODUCTION}

Parkinsonism is diagnosed when a patient manifests three cardinal motor symptoms, bradykinesia in combination with either resting tremor, rigidity, or both. ${ }^{1)}$ Parkinson's disease (PD) is the most common cause of parkinsonism, and parkinsonism can result from neurodegenerative, vascular, drug-related, infectious, toxic, and other unknown secondary causes. ${ }^{2)}$ According to the Europarkinson collaborative study, the prevalence of parkinsonism was $2.3 \%$, whereas the prevalence of PD was $1.6 \% .{ }^{3)}$ One study reported the crude prevalence of PD to be $0.42 \%$ in Korea. ${ }^{4)}$ As the disease progresses, cognitive impairment, neuropsychiatric changes, and autonomic-nervous system failure develops in patients with parkinsonism in addition to the exacerbation of classic motor symptoms. ${ }^{5)}$ These changes eventually increase economic and caregiver burden of family members; thus, the role of family members becomes crucial in controlling the disease. ${ }^{6,7)}$

Several studies have reported that family dynamics and diseases influence each other ${ }^{8,9)}$ Family dynamics are especially important in diseases that cause a considerable burden on family members because they can affect caregiver burden in addition to the disease itself. In families of persons with aphasia, a high level of communication selfefficacy was associated with a low burden of family communication and good mental health. ${ }^{10)}$ In a cross-sectional study examining the family dynamics of caregivers for patients with dementia, family empathy and general functioning were associated with the quality of care provided by informal caregivers. ${ }^{8)}$ In previous studies, a significantly higher frequency of obsessive-compulsive personality disorder was reported in patients with parkinsonism, and depression and anxiety were reported as comorbidities of PD. ${ }^{11,12)}$ These neuropsychiatric changes in patients with parkinsonism may worsen family functioning and communication.

According to the Family Adaptability and Cohesion Evaluation Scale (FACES), a family's functioning can be determined by evaluating its adaptability and cohesion. ${ }^{13)}$ Adaptability refers to a family's ability to change its role relationships, power structure, and rules in response to a situation, whereas cohesion refers to the emotional bonding that exists between family members. ${ }^{13,14)}$ Family communication facilitates these two dimensions of family functioning. ${ }^{13,15)}$

As previously stated, several studies have reported family dynamics to be associated with diseases and to influence caregiver burden., ${ }^{8,16)}$ However, studies focusing on family dynamics in spouses are rare, especially in East Asia. It is essential to assess the family dynamics of spouses of patients with parkinsonism because they are often the main informal) caregivers for the patients. Results from a previous study indicated that a husband's alcohol consumption had negative effects on his wife's family communication. ${ }^{17)}$ In this study, we investigated the effects of spouses' parkinsonism on family functioning and communication using data from a cohort of couples who visited primary care practices in Korea.

\section{METHODS}

\section{Study Participants}

This study was based on the data collected for the Family Cohort Study in Primary Care. The study's protocol and the written informed consent form were approved by the institutional review board of Asan Medical Center (IRB No. 2010-0370). The participants read and signed the informed consent form prior to the beginning of the study. Study participants without parkinsonism were consecutively enrolled by 28 family physicians from 22 hospitals between April 2009 and June 2011. Couples who visited hospital-based family practices for the treatment of chronic diseases were consecutively enrolled, and these patients comprised the study's control group. Patients with parkinsonism and their spouses were recruited from a single institution. Finally, a total of 1,164 study participants (582 couples) were enrolled. Among the 582 couples, there were 65 couples with parkinsonism and 517 couples in the control group. For the data analysis, we selected 65 spouses of the patients with parkinsonism and either a husband or a wife from the 517 couples in the control group; therefore, a total of 582 participants were included in the analyses. When selecting either a husband or a wife from the 517 couples, we alternated in the selection a husband or a wife according to their order of enrollment.

\section{Data Collection and Measurement}

All of the physicians collected data using standardized questionnaires, which included questions regarding demographic characteristics such as age, sex, educational level, and income, as well as lifestyle factors, such as smoking habits, alcohol intake, and physical activity. All of the questionnaires used in this study were self-administered. Educational level was categorized into three groups: $\leq 12$ years, $13-16$ years, and $>16$ years. Monthly income was divided into four categories: $<2.00$ million won, 2.00-3.99 million won, 4.00-5.99 million won, and $\geq 6.00$ million won. Smoking status was classified as nonsmoker, ex-smoker, and current smoker, and alcohol consumption was categorized in terms of the number of glasses consumed per week. Physical activity was measured using the International Physical Activity Questionnaire ShortForm, which is a scale based on activity intensity and exercise time during the previous 7 days. ${ }^{18)}$ Physical activity was classified as low, moderate, and high. The high activity level was defined as the achievement of 1,500 metabolic equivalent (MET)-min/wk with $\geq 3$ days of activity of vigorous-intensity, or 3,000 MET-min/wk with 7 days of any combination of walking, activity of moderate intensity, or of vigorousintensity. Moderate activity was defined as performance of vigorous activity for $\geq 20 \mathrm{~min} / \mathrm{d}$ for $\geq 3$ days or activity of moderate intensity, or walking for $\geq 30 \mathrm{~min} / \mathrm{d}$ for $\geq 5$ days or achieving 600 MET-min/wk with $\geq 5$ days of any combination of walking, activity of moderate intensity or of vigorous intensity. Low activity was defined as not meeting the criteria for either high or moderate activity. Height and body weight were measured, and body mass index (BMI) was calculated as body weight divided by the square of the height $\left(\mathrm{kg} / \mathrm{m}^{2}\right)$. BMI was categorized into 4 groups, and the cut-off values for overweight, first degree 
obesity, and second degree obesity were $23.0,25.0$, and $30.0 \mathrm{~kg} / \mathrm{m}^{2}$, respectively, according to the Asia-Pacific Classification. ${ }^{19)}$ Abdominal obesity for Korean men and women was defined as a waist circumference $\geq 90$ and $\geq 85 \mathrm{~cm}$, respectively. ${ }^{20)}$ In addition, information about previous medical history and concomitant medications was collected.

\section{Definition of Parkinsonism}

In this study, parkinsonism was defined as: (1) receiving a diagnosis from the Korean Classification of Diseases beginning with a diagnostic code of G20 or G21, and (2) taking prescribed medication for symp- toms of parkinsonism. A professional neurologist diagnosed all of the patients, and the standardized questionnaires and medical records of the spouses of patients with parkinsonism were reviewed for analysis.

\section{Measurement of Family Functioning}

Family functioning was evaluated using the Korean version of the FACES-III, which was developed by Olson and colleagues to measure the quality of family relationships. It consists of 10 questions assessing family adaptability and 10 questions assessing family cohesion. ${ }^{21)}$ Each question is scored on a scale ranging from 1 to 5 . The total score is

Table 1. Basic characteristics of 582 study participants

\begin{tabular}{|c|c|c|c|c|}
\hline Characteristic & Total $(N=582)$ & Spouses of patients with parkinsonism $(n=65)$ & Spouses of patients without parkinsonism $(n=517$ ) & P-value \\
\hline Age $(y)$ & & & & $<0.001$ \\
\hline$<50$ & $98(18.49)$ & $8(12.90)$ & $90(19.23)$ & \\
\hline $50-59$ & $176(33.21)$ & $12(19.35)$ & $164(35.04)$ & \\
\hline $60-69$ & 205 (38.68) & $28(45.16)$ & $177(37.82)$ & \\
\hline$\geq 70$ & $51(9.62)$ & $14(22.58)$ & $37(7.91)$ & \\
\hline Sex & & & & 1.000 \\
\hline Male & $291(50.00)$ & $32(49.23)$ & $259(50.10)$ & \\
\hline Female & $291(50.00)$ & $33(50.77)$ & $258(49.90)$ & \\
\hline Education (y) & & & & 0.228 \\
\hline$\leq 12$ & $323(55.88)$ & $42(65.63)$ & $281(54.67)$ & \\
\hline $13-16$ & $196(33.91)$ & $16(25.00)$ & 180 (35.02) & \\
\hline$>16$ & 59 (10.21) & $6(9.38)$ & 53 (10.31) & \\
\hline Income (10,000 won/mo) & & & & $<0.001$ \\
\hline$<200$ & 120 (21.39) & $30(47.62)$ & 90 (18.07) & \\
\hline 200-399 & 168 (29.95) & 17 (26.98) & 151 (30.32) & \\
\hline $400-599$ & $122(21.75)$ & $9(14.29)$ & $113(22.69)$ & \\
\hline$\geq 600$ & $151(26.92)$ & $7(11.11)$ & 144 (28.92) & \\
\hline Smoking status & & & & 0.341 \\
\hline Nonsmoker & 283 (54.32) & 35 (57.38) & 248 (53.91) & \\
\hline Ex-smoker & $166(31.86)$ & $15(24.59)$ & $151(32.83)$ & \\
\hline Current smoker & $72(13.82)$ & $11(18.03)$ & $61(13.26)$ & \\
\hline Amount of alcohol drinking & & & & 0.678 \\
\hline$<1$ glasses/wk & 289 (53.92) & 32 (53.33) & 257 (53.99) & \\
\hline 1-14 glasses/wk & 157 (29.29) & $19(31.67)$ & 138 (28.99) & \\
\hline 15-35 glasses/wk & 65 (12.13) & $8(13.33)$ & 57 (11.97) & \\
\hline$\geq 36$ glasses/wk & $25(4.66)$ & $1(1.67)$ & $24(5.04)$ & \\
\hline Physical activity & & & & 0.127 \\
\hline Low & 162 (29.89) & $14(22.58)$ & $148(30.83)$ & \\
\hline Moderate & $180(33.21)$ & $18(29.03)$ & $162(33.75)$ & \\
\hline High & $200(36.90)$ & $30(48.39)$ & 170 (35.42) & \\
\hline Body mass index $\left(\mathrm{kg} / \mathrm{m}^{2}\right)$ & & & & 0.094 \\
\hline$<23.0$ & $172(33.08)$ & $28(43.75)$ & $144(31.58)$ & \\
\hline 23.0-24.9 & 159 (30.58) & $15(23.44)$ & $144(31.58)$ & \\
\hline $25.0-29.9$ & 170 (32.69) & $21(32.81)$ & 149 (32.68) & \\
\hline$\geq 30$ & 19 (3.65) & 0 & $19(4.17)$ & \\
\hline Waist circumference & & & & 0.886 \\
\hline Normal & $305(65.87)$ & $40(64.52)$ & 265 (66.08) & \\
\hline Abdominal obesity & $158(34.13)$ & $22(35.48)$ & 136 (33.92) & \\
\hline \multicolumn{5}{|l|}{ Comorbidities } \\
\hline Cerebrovascular disease & $36(6.33)$ & $9(13.85)$ & 27 (5.36) & 0.014 \\
\hline Depression & $25(4.39)$ & $4(6.15)$ & $21(4.17)$ & 0.514 \\
\hline Cancer & $21(3.70)$ & $2(3.10)$ & $19(3.80)$ & 1.000 \\
\hline
\end{tabular}

Values are presented as number (\%). Pearson's chi-square test was used to compare the characteristics of the spouses with and without parkinsonism. Missing data were excluded. 
based on the sum of the respondent's points on the scale. Family adaptability is classified as rigid (10-19 patients), structured (20-24 patients), flexible (25-28 patients), and chaotic (29-50 patients), and family cohesion is classified as disengaged (10-35 patients), separated (36-40 patients), connected (41-45 patients), and enmeshed (46-50 patients). Family functioning is defined as the combined categories of family adaptability and cohesion and is classified as balanced, midrange, and extreme family types.

\section{Measurement of Family Communication}

Family communication was evaluated using the Korean version of the Family Communication Scale (FCS) of the FACES-IV. ${ }^{15,22)}$ The FCS consists of 10 questions, with each question scored from 1 to 5 . According to the sum of the scores, family communication is classified as low (10-35), moderate (36-39), and high (40-50). Higher scores represent better family communication.

\section{Statistical Analyses}

The initial analyses of the data focused on descriptive statistics. Student t-test was used to compare family adaptability, cohesion, and FCS scores of the spouses of patients with and without parkinsonism. Pearson's chi-square test was used to compare the characteristics, family functioning, and family communication between the two groups of spouses. Multinomial logistic regression models were used to estimate the odds ratios (ORs) and 95\% confidence intervals (CIs) of each level of family functioning and communication in association with the presence of spousal parkinsonism. Multivariate logistic regression was performed after adjusting for potential confounders identified by the chi-square test, such as age, sex, income, and cerebrovascular disease. A crude analysis was performed in model 1. In model 2, we adjusted for age and sex, which were the main demographic factors. In model 3, we also adjusted for income, which was a significant demographic confounder according to the chi-square test. Finally, we added cerebrovascular disease as a covariate to model 4 because it was significantly different between the spouses of patients with and without parkinsonism. In this study, $\mathrm{P}<0.05$ was considered statistically significant. All data were analyzed using Stata ver. 14.0 for Windows (Stata Co., College Station, TX, USA).

\section{RESULTS}

\section{Basic Characteristics of the Study Participants}

Table 1 lists the basic characteristics of the study participants. Their mean age \pm standard deviation was $57.9 \pm 9.6$ years and most of them (71.9\%) were between 50 and 70 years of age. Fifty percent of the participants were male, and $55.9 \%$ had $\leq 12$ years of education. Approximately $13.8 \%$ of the participants were current smokers and $16.8 \%$ drank more than 15 glasses of alcohol per week. The mean BMI \pm standard deviation was $24.3 \pm 3.05 \mathrm{~kg} / \mathrm{m}^{2} ; 36.3 \%$ of the participants were obese (BMI $\geq 25 \mathrm{~kg} / \mathrm{m}^{2}$ ), and $34.1 \%$ had abdominal obesity. Cerebrovascular disease was present in $6.33 \%$ of the participants, and depression and cancer were present in $4.39 \%$ and $3.70 \%$ of the participants, respectively. The chi-square test results for demographic factors indicated that age and income were significantly different between the spouses with and without parkinsonism (Table 1). Among the comorbidities, cerebrovascular disease showed a significant difference.

\section{Association between Spousal Parkinsonism and Family Functioning}

Tables 2 and 3 present the differences in the family adaptability and cohesion scores between the spouses of patients with and without parkinsonism. The mean family adaptability and cohesion scores of the spouses of patients with parkinsonism were 23.09 and 32.40, respectively, whereas those of the control group were 23.84 and 34.89, respectively. There was no significant difference in the family adaptability scores $(\mathrm{P}=0.372)$; however, a significant difference was found in the family cohesion scores ( $\mathrm{P}=0.022)$ (Table 2$)$. Altogether, there was a significant difference in family functioning between the spouses of patients with and without parkinsonism $(\mathrm{P}=0.014)$ (Table 3). The ORs for family functioning according to the presence of spousal parkinsonism are presented in Table 4 . The univariate multinomial analysis showed that the OR for midrange family functioning did not increase significantly compared with the OR for balanced family functioning; however, the OR for extreme family functioning increased significantly compared with balanced family functioning when the two groups of spouses were compared (OR, 2.11; 95\% CI, 0.84 to 5.30 for midrange functioning and OR, 3.74; $95 \% \mathrm{CI}, 1.44$ to 9.69 for extreme functioning). These results were consistent with those of the multivariate multinomial analyses. Model 2 was adjusted for age and sex, and model 3 was adjusted for age, sex, and income. In model 4 , when all possible confounders including age, sex, income, and cardiovascular disease

Table 2. Differences in mean scores on family adaptability, cohesion, and the FCS between the spouses of patients with and without parkinsonism

\begin{tabular}{|c|c|c|c|c|c|c|c|c|}
\hline & \multicolumn{3}{|c|}{ Spouses of patients with parkinsonism $(n=65)$} & \multicolumn{3}{|c|}{ Spouses of patients without parkinsonism $(n=517)$} & \multirow{2}{*}{ t-value } & \multirow{2}{*}{ P-value } \\
\hline & Mean & $(95 \% \mathrm{Cl})$ & $\mathrm{SD}$ & Mean & $(95 \% \mathrm{Cl})$ & SD & & \\
\hline Adaptability score & 23.09 & $(21.51-24.67)$ & 6.48 & 23.84 & (23.33-24.35) & 5.88 & -0.89 & 0.372 \\
\hline Cohesion score & 32.40 & $(30.35-34.45)$ & 8.43 & 34.89 & (34.24-35.54) & 7.59 & -2.31 & 0.022 \\
\hline FCS score & 36.13 & (34.30-37.96) & 7.54 & 38.59 & (38.00-39.18) & 6.79 & -2.66 & 0.008 \\
\hline
\end{tabular}

Student t-test was used to compare the mean scores representing family dynamics between the two groups.

FCS, Family Communication Scale; Cl, confidence interval; SD, standard deviation. 
Table 3. Differences in family functioning and communication between the spouses of the patients with and without parkinsonism

\begin{tabular}{|c|c|c|c|c|}
\hline & Total $(\mathrm{N}=582)$ & Spouses of patients with parkinsonism $(n=65)$ & Spouses of patients without parkinsonism $(n=517)$ & P-value \\
\hline Adaptability & & & & 0.323 \\
\hline Rigid & 124 (24.65) & $16(28.57)$ & $108(24.16)$ & \\
\hline Structured & $153(30.42)$ & $18(32.14)$ & $135(30.20)$ & \\
\hline Flexible & $131(26.04)$ & $9(16.07)$ & $122(27.29)$ & \\
\hline Chaotic & 95 (18.89) & $13(23.21)$ & 82 (18.34) & \\
\hline Cohesion & & & & 0.029 \\
\hline Disengaged & 271 (51.23) & 37 (64.91) & 234 (49.58) & \\
\hline Separated & $139(26.28)$ & $13(22.81)$ & $126(26.69)$ & \\
\hline Connected & $83(15.69)$ & $2(3.51)$ & $81(17.16)$ & \\
\hline Enmeshed & $36(6.81)$ & $5(8.77)$ & $31(6.57)$ & \\
\hline Family functioning & & & & 0.014 \\
\hline Balanced & $116(24.37)$ & $6(11.76)$ & $110(25.88)$ & \\
\hline Midrange & $242(50.84)$ & $25(49.02)$ & $217(51.06)$ & \\
\hline Extreme & $118(24.79)$ & $20(39.22)$ & $98(23.06)$ & \\
\hline Family communication & & & & 0.001 \\
\hline High & $256(46.04)$ & $15(24.19)$ & 241 (48.79) & \\
\hline Moderate & $160(28.78)$ & $28(45.16)$ & 132 (26.72) & \\
\hline Low & $140(25.18)$ & $19(30.65)$ & $121(24.49)$ & \\
\hline
\end{tabular}

Values are presented as number (\%). Pearson's chi-square test was used to compare the family functioning and family communication of the spouses with and without parkinsonism. Missing data were excluded.

Table 4. Multinomial logistic regression analysis of the associations between spousal parkinsonism and family dynamics

\begin{tabular}{|c|c|c|c|c|}
\hline & \multicolumn{4}{|c|}{ Spouses of patients with parkinsonism in comparison to the control group* } \\
\hline & Model 1 & Model 2 & Model 3 & Model 4 \\
\hline \multicolumn{5}{|c|}{ Family functioning } \\
\hline Balanced & 1.00 & 1.00 & 1.00 & 1.00 \\
\hline Midrange & $2.11(0.84-5.30)$ & $1.91(0.75-4.88)$ & $1.89(0.74-4.87)$ & $1.82(0.71-4.70)$ \\
\hline Extreme & $3.74(1.44-9.69)$ & $3.36(1.28-8.85)$ & $3.46(1.29-9.25)$ & $3.45(1.28-9.28)$ \\
\hline \multicolumn{5}{|c|}{ Family communication } \\
\hline High & 1.00 & 1.00 & 1.00 & 1.00 \\
\hline Moderate & 3.41 (1.76-6.61) & $3.62(1.82-7.19)$ & 3.81 (1.88-7.72) & $3.85(1.89-7.84)$ \\
\hline Low & $2.52(1.24-5.14)$ & $2.72(1.31-5.68)$ & $2.23(1.05-4.74)$ & $2.21(1.03-4.72)$ \\
\hline
\end{tabular}

Values are presented as odds ratio (95\% confidence interval). Model 1: crude; model 2: adjusted for age and sex; model 3: adjusted for age, sex, and income; model 4: adjusted for age, sex, income, and cerebrovascular disease.

*The control group refers to the spouses of the patients without parkinsonism.

were adjusted, the OR for extreme family functioning remained significant between the spouses of patients with and without parkinsonism (OR, 1.82; 95\% CI, 0.71 to 4.70 for midrange functioning and OR, 3.45; $95 \% \mathrm{CI}, 1.28$ to 9.28 for extreme functioning).

\section{Association between Spousal Parkinsonism and Family Communication}

Tables 2 and 3 present differences in family communication between the spouses of the patients with and without parkinsonism. The mean FCS score of the spouses of patients with parkinsonism was 36.13, whereas that of the control group was 38.59 (Table 2). There was significant difference in family communication between the spouses of the patients with and without parkinsonism ( $\mathrm{P}=0.001)$ (Table 3). The ORs for family communication according to the presence of spousal parkinsonism are presented in Table 4. The univariate multinomial analysis showed that the OR for moderate family communication in- creased significantly as compared with high family communication when comparing the spouses of the patients with parkinsonism to the control group (OR, 3.41; 95\% CI, 1.76 to 6.61 ). In addition, the OR for low-family communication significantly increased in comparison with high-family communication group (OR, 2.52; 95\% CI, 1.24 to 5.14). These results were consistent with those of the multivariate multinomial analyses. In model 4, when all possible confounders, including age, sex, income, and cardiovascular disease were adjusted, the OR for moderate- and low-family communication remained significant when comparing the spouses of the patients with parkinsonism to the control group (OR, 3.85: 95\% CI 1.89 to 7.84 for moderate communication and $\mathrm{OR}, 2.21$; $95 \% \mathrm{CI}, 1.03$ to 4.72 for low communication).

\section{DISCUSSION}

This study found a deterioration in family functioning and family com- 
munication in spouses of patients with parkinsonism. Family functioning tended to be more extreme, and family communication was significantly poorer in spouses of patients with parkinsonism in comparison to the spouses in the control group. To the best of our knowledge, this is the first study evaluating the effects of spousal parkinsonism on family functioning and communication.

Family functioning and communication in spouses of patients with parkinsonism were significantly worse compared with spouses of patients without parkinsonism. Family dynamics can be aggravated because of the cognitive impairment and neuropsychiatric changes that develop in parkinsonism. ${ }^{5)}$ Previous studies have reported aggravated family dynamics when cognition is impaired due to various causes. In a systemic review investigating the impact of traumatic brain injury (TBI) on family functioning, there was a trend in families whose child had a severe TBI for greater dysfunction. ${ }^{23)}$ Furthermore, Wiguna et $\mathrm{al}^{24)}$ reported that unhealthy parental relationships were observed in parents who had a child with schizophrenia compared to parents who did not have a child with schizophrenia. Neuropsychiatric changes, such as personality changes and mood instability, can also influence family dynamics. In a cross-sectional study comparing family functioning in families of depressed patients and nonclinical control families, the family functioning of a person with a depressed family member was more likely to be poorer than a person in the control group. ${ }^{16)}$ Patients with parkinsonism manifest cognitive impairment, and neuropsychiatric changes, including personality changes, depressed mood, and anxiety, which can negatively affect family functioning. ${ }^{11,12)}$ In addition, symptoms such as masked facial expression, blurred vision, and speech impairment can make it difficult for the affected family member to communicate. ${ }^{5)}$ Regarding communication disorders, dysarthria makes conversation most difficult; however, other linguistic and cognitive difficulties become apparent in the later stages of the disease. Partners overcome communication difficulties by using various strategies, such as guessing and requesting repetition and elaboration. ${ }^{25)}$

Symptoms of parkinsonism increase caregiver burden and increased burden can ultimately lead to aggravated family dynamics. In previous studies, diseases that cause burden on family members influenced family dynamics. In a cross-sectional study examining the family dynamics of caregivers for persons with dementia, family dynamics were significantly associated with caregiver depression, stress, satisfaction with life, and burden. ${ }^{9}$ In families with a patient diagnosed with a debilitating neurodegenerative disease, such as amyotrophic lateral sclerosis, patterns of extreme family functioning were observed. ${ }^{26)}$ In PD patients, disability and mood were reported as main factors contributing to caregiver burden, and neuropsychiatric symptoms, such as depression, anxiety, and mental fatigue were associated with caregiver burden. ${ }^{7,27)}$ Caring for a chronically ill spouse is stressful; however, partners are usually the main informal caregiver of patients with parkinsonism. Unremitting stress in the environment might aggravate family dynamics in spouses of patients with parkinsonism.

In this study, we used the Korean version of the FACES-III to assess family functioning and the FCS of the FACES-IV to evaluate family communication. The FACES was initially used to measure the quality of children's family relations; however, it has also been used to evaluate family dynamics in spouses and has demonstrated efficacy. ${ }^{28,29)}$ In a systemic review of family assessment measures, the FACES was found to be suitable for clinical use. ${ }^{30)}$

The strength of this study is that we evaluated the family dynamics of couples with parkinsonism in comparison to couples without parkinsonism who visited hospital-based primary clinics. Therefore, this study demonstrated how neurodegenerative diseases, such as parkinsonism, influence family functioning and communication compared with common chronic diseases. To the best of our knowledge, this study is the first to report the relationship between family functioning and spousal parkinsonism in the Korean population. Although several studies have reported dysfunctional dynamics in families with patients with comorbidities, none of them found poorer family functioning and communication in spouses of patients with parkinsonism. Primary care physicians may encounter patients with neurodegenerative disorders because those patients also seek treatment for diseases, such as hypertension, diabetes, and dyslipidemia. These physicians should consider the deterioration of the family's dynamics and the burden of its members to provide family-oriented medical service. The results of this study provided new aspects of the family dynamics of patients with parkinsonism.

There are several limitations in our study. Although the number of patients in our sample was large, most of them actually comprised the control group, whereas only 65 of them were spouses of patients with parkinsonism. This may have led to a conclusion that would have been different if both groups of participants had been equally distributed. In addition, participants were patients visiting hospital-based primary clinics and the department of neurology; thus, this group does not represent the general population. We adjusted for age and income in our multinomial regression analyses because these factors showed significant differences between the two groups. However, instead of adjusting for possible confounders, we could have selected matched samples during the process of sample selection. Furthermore, we did not consider the quality of life of the spouses and the severity of parkinsonism among the patients. Quality of life and severity of disease are especially important in neurodegenerative disorders, and both variables have been associated with caregiver burden and family dynamics. There are tools that specifically measure the quality of life and severity of PD; however, we did not take this into consideration. These variables may be possible confounders in the assessment of the relationship between family functioning and spousal parkinsonism. Thus, future studies should focus on measuring quality of life and disease severity when investigating family dynamics in neurodegenerative disorders.

In conclusion, family functioning and communication deteriorated in the group of spouses of patients with parkinsonism. Patients with parkinsonism develop cognitive impairments and neuropsychiatric changes, and parkinsonism had a negative association with the family dynamics of the spouses in this study. Counseling to improve family 
functioning and communication will be needed by families of patients with parkinsonism to relieve caregiver burden and maintain healthy relationships over the long-term course of the disease. In particular, primary physicians should consider the family dynamics of each patient to provide family-oriented medical service.

\section{CONFLICT OF INTEREST}

No potential conflict of interest relevant to this article was reported.

\section{REFERENCES}

1. Postuma RB, Berg D, Stern M, Poewe W, Olanow CW, Oertel W, et al. MDS clinical diagnostic criteria for Parkinson's disease. Mov Disord 2015;30:1591-601.

2. Christine CW, Aminoff MJ. Clinical differentiation of parkinsonian syndromes: prognostic and therapeutic relevance. Am J Med 2004;117:412-9.

3. De Rijk MC, Tzourio C, Breteler MM, Dartigues JF, Amaducci L, LopezPousa S, et al. Prevalence of parkinsonism and Parkinson's disease in Europe: the EUROPARKINSON Collaborative Study. European Community Concerted Action on the Epidemiology of Parkinson's disease. J Neurol Neurosurg Psychiatry 1997;62:10-5.

4. Kim JM, Kim JS, Kim KW, Lee SB, Park JH, Lee JJ, et al. Study of the prevalence of Parkinson's disease using dopamine transporter imaging. Neurol Res 2010;32:845-51.

5. Beitz JM. Parkinson's disease: a review. Front Biosci (Schol Ed) 2014;6:65-74.

6. Findley LJ. The economic impact of Parkinson's disease. Parkinsonism Relat Disord 2007;13 Suppl:S8-S12.

7. Martinez-Martin P, Rodriguez-Blazquez C, Forjaz MJ, Frades-Payo B, Aguera-Ortiz L, Weintraub D, et al. Neuropsychiatric symptoms and caregiver's burden in Parkinson's disease. Parkinsonism Relat Disord 2015;21:629-34.

8. Panyavin I, Trujillo MA, Peralta SV, Stolfi ME, Morelli E, Perrin PB, et al. Examining the influence of family dynamics on quality of care by informal caregivers of patients with Alzheimer's dementia in Argentina. Am J Alzheimers Dis Other Demen 2015;30:613-21.

9. Sutter M, Perrin PB, Chang YP, Hoyos GR, Buraye JA, Arango-Lasprilla JC. Linking family dynamics and the mental health of Colombian dementia caregivers. Am J Alzheimers Dis Other Demen 2014;29:67-75.

10. Tatsumi H, Nakaaki S, Satoh M, Yamamoto M, Chino N, Hadano K. Relationships among communication self-efficacy, communication burden, and the mental health of the families of persons with aphasia. J Stroke Cerebrovasc Dis 2016;25:197-205.

11. Nicoletti A, Luca A, Luca M, Mostile G, Sciacca G, Petralia A, et al. Obsessive-compulsive personality disorder in drug-naive Parkinson's disease patients. J Neurol 2015;262:485-6.

12. Wee N, Kandiah N, Acharyya S, Chander RJ, Ng A, Au WL, et al. Depression and anxiety are co-morbid but dissociable in mild Parkinson's disease: a prospective longitudinal study of patterns and predictors. Parkinsonism Relat Disord 2016;23:50-6.

13. Olson DH. Family assessment and intervention: the circumplex model of family systems. Child Youth Serv 1989;11:9-48.

14. Place M, Hulsmeier J, Brownrigg A, Soulsby A. The Family Adaptability and Cohesion Evaluation Scale (FACES): an instrument worthy of rehabilitation? Psychiatrist 2005;29:215-8.

15. Franklin C, Streeter CL, Springer DW. Validity of the FACES IV family assessment measure. Res Soc Work Pract 2001;11:576-96.

16. Du N, Ran MS, Liang SG, SiTu MJ, Huang Y, Mansfield AK, et al. Comparison of family functioning in families of depressed patients and nonclinical control families in China using the Family Assessment Device and the Family Adaptability and Cohesion Evaluation Scales II. Ann Clin Psychiatry 2014;26:47-56.

17. Kwon HJ, Ahn TK, Lee JA, Sunwoo S, Kim YS, Kim BS, et al. The relationship between a spouse's alcohol use disorder and family communication. Korean J Fam Med 2015;36:92-102.

18. IPAQ Research Committee. Guidelines for data processing and analysis of the international physical activity questionnaire (IPAQ): short and long forms [Internet]. [place unknown]: IPAQ Research Committee; 2005 [cited 2006 Jan 16]. Available from: http://www.ipaq.ki.se.

19. World Health Organization, International Association for the Study of Obesity, International Obesity Task Force. The Asia-Pacific perspective: redefining obesity and its treatment. Sydney: Health Communications; 2000.

20. Lee SY, Park HS, Kim DJ, Han JH, Kim SM, Cho GJ, et al. Appropriate waist circumference cutoff points for central obesity in Korean adults. Diabetes Res Clin Pract 2007;75:72-80.

21. Lim JH, Lee KR, Oh MK, Kwak KW, Yoon BB. A study of reliability and validity of FACES III. J Korean Acad Fam Med 1990;11:8-17.

22. Kim YS, Sunwoo S, Kim B, Park HK, Ok SW, Cha D. Reliability and validity of Family Communication Scale in the FACES IV package: Korean version. J Korean Fam Relat Assoc 2012;17:241-58.

23. Rashid M, Goez HR, Mabood N, Damanhoury S, Yager JY, Joyce AS, et al. The impact of pediatric traumatic brain injury (TBI) on family functioning: a systematic review. J Pediatr Rehabil Med 2014;7:241-54.

24. Wiguna T, Ismail RI, Noorhana SR, Kaligis F, Aji AN, Belfer ML. Family responses to a child with schizophrenia: An Indonesian experience. Asian J Psychiatr 2015;18:66-9.

25. Carlsson E, Hartelius L, Saldert C. Communicative strategies used by spouses of individuals with communication disorders related to stroke-induced aphasia and Parkinson's disease. Int J Lang Commun Disord 2014;49:722-35.

26. Tramonti F, Barsanti I, Bongioanni P, Bogliolo C, Rossi B. A permanent emergency: a longitudinal study on families coping with amyotrophic lateral sclerosis. Fam Syst Health 2014;32:271-9.

27. Santos-Garcia D, de la Fuente-Fernandez R. Factors contributing to caregivers' stress and burden in Parkinson's disease. Acta Neurol Scand 2015;131:203-10.

28. Henggeler SW, Burr-Harris AW, Borduin CM, McCallum G. Use of the Family Adaptability and Cohesion Evaluation Scales in child clinical research. J Abnorm Child Psychol 1991;19:53-63.

29. Finzi-Dottan R, Cohen O, Iwaniec D, Sapir Y, Weizman A. The druguser husband and his wife: attachment styles, family cohesion, and adaptability. Subst Use Misuse 2003;38:271-92.

30. Hamilton E, Carr A. Systematic review of self-report family assessment measures. Fam Process 2016;55:16-30. 